\title{
Simple method for any planar wiggler field simulation
}

\author{
M. N. Smolyakov \\ Physical Department, Moscow State University, Vorob'evy Gory, 119899 Moscow, Russia
}

(Received 29 September 2000; published 25 April 2001)

\begin{abstract}
This paper deals with a nonstandard method for calculating the magnetic field of planar wigglers and undulators consisting of pure permanent magnets. This method of calculation is based on certain properties of the Fourier transform. It allows the analytical expression of the Fourier transform for the planar magnetic fields through the wiggler's geometry and magnetization of its blocks. The upper theoretical limit for the amplitude of the magnetic field is derived and matched with the field amplitude of planar wigglers with standard designs. The property of universality for planar wigglers is also taken into consideration as it may greatly simplify the analysis of magnetic fields for wigglers with different designs.
\end{abstract}

DOI: 10.1103/PhysRevSTAB.4.040701

PACS numbers: 41.60.- m, 75.50.Ww, 07.85.Qe

\section{INTRODUCTION}

Presently, permanent magnets are widely used in wiggler and undulator designs. Microundulators often employ nonstandard schemes based on permanent magnets as well. The importance played by permanent magnets generates a need for the thorough study of the wiggler's magnetic field, concentrating principally on its general properties. The straightforward way of analyzing the magnetic field properties is to calculate analytically (or numerically) the magnetic field distribution using the following analysis of its characteristics. In particular, the easy axis rotation theorem has been proved for planar magnetic systems: if at each point of a pure permanent magnet system all of its easy axes are rotated by an angle $\theta$ while the system geometry remains the same, then all magnetic fields outside the magnetic system rotate by the opposite angle $-\theta$ without a change in amplitude [1]. If this is true, the vertical component of the magnetic field is changed, producing corresponding changes in the radiation spectra and power. The easy axis symmetry principle, which is valid in three dimensions, was also considered and used in the magnetic field analysis for wigglers [2].

This paper deals with an alternative method for planar (two-dimensional) magnetic field analysis through the direct computation of its Fourier transform. The Fourier transform of planar magnetic fields may be expressed analytically in terms of the magnetic system's geometry and the distribution of its magnetization, thus describing explicitly their effects on the magnetic field. This method provides possible proof for the property of universality for planar wigglers: if all easy axes of the wiggler's top part are rotated by the angle $\theta$, and at the same time all easy axes of its bottom part are rotated by the opposite angle $-\theta$ while keeping the system geometry the same, the magnetic field varies, while the absolute value of the Fourier transform of the magnetic field remains the same. From this it follows that the total power of wiggler radiation, as well as the positions of radiation harmonics, remains unchanged under such modification of the wiggler's magnetization. This paper also deals with the upper theoretical limit for the magnetic field amplitude of planar pure permanent magnet wigglers. The value of this limit is matched with that of standard wigglers. The method proposed herein can considerably simplify the computer simulation and analysis of magnetic fields for planar wigglers with different designs.

\section{MAGNETIC FIELD SIMULATION}

Let us consider a magnetic system with the magnetic moment distribution $\vec{M}\left(\vec{r}^{\prime}\right)$. The magnetic field $\vec{B}(\vec{r})$, generated by this system outside the magnets, is given by the following expression [3]:

$$
\vec{B}(\vec{r})=\int d^{3} \vec{r}^{\prime} \frac{3 \vec{R}\left(\vec{M}\left(\vec{r}^{\prime}\right) \cdot \vec{R}\right)-\vec{M}\left(\vec{r}^{\prime}\right) \cdot R^{2}}{R^{5}},
$$

where $\vec{r}=(x, y, z), \vec{r}^{\prime}=\left(x^{\prime}, y^{\prime}, z^{\prime}\right), \vec{R}=\vec{r}-\vec{r}^{\prime}, R=$ $|\vec{R}|$, and the integration is taken over the volume of magnets. We use here the right-hand Cartesian system of coordinates with the $x$ and $y$ axis directed horizontally and the $z$ axis directed upwards. The xoy plane is assumed to be free of the magnetic material. Let us next imagine that the magnetic system is infinitely wide and homogeneous along the $x$ axis. By this it is meant that the magnetic moment $\vec{M}\left(\vec{r}^{\prime}\right)$ is $x$ independent: $\vec{M}\left(\vec{r}^{\prime}\right)=\left[0, M_{y}\left(y^{\prime}, z^{\prime}\right), M_{z}\left(y^{\prime}, z^{\prime}\right)\right]$. If this is true, we may perform the integration along the $x$ axis in Eq. (1) and express the field through the integrals over the cross section of the magnetic system:

$$
\begin{aligned}
& B_{y}(y, z)=\int d y^{\prime} d z^{\prime} \frac{2 M_{y}\left(y^{\prime}, z^{\prime}\right)\left[\left(y-y^{\prime}\right)^{2}-\left(z-z^{\prime}\right)^{2}+4 M_{z}\left(y^{\prime}, z^{\prime}\right)\left(y-y^{\prime}\right)\left(z-z^{\prime}\right)\right]}{\left[\left(y-y^{\prime}\right)^{2}+\left(z-z^{\prime}\right)^{2}\right]^{2}}, \\
& B_{z}(y, z)=\int d y^{\prime} d z^{\prime} \frac{-2 M_{z}\left(y^{\prime}, z^{\prime}\right)\left[\left(y-y^{\prime}\right)^{2}-\left(z-z^{\prime}\right)^{2}+4 M_{y}\left(y^{\prime}, z^{\prime}\right)\left(y-y^{\prime}\right)\left(z-z^{\prime}\right)\right]}{\left[\left(y-y^{\prime}\right)^{2}+\left(z-z^{\prime}\right)^{2}\right]^{2}} .
\end{aligned}
$$


The easy axis rotation theorem may be derived easily from expressions (2) and (3). Let us turn the magnetic moments through the angle $\theta$ in an anticlockwise direction:

$$
\begin{aligned}
& M_{y}^{\prime}(y, z)=M_{y}(y, z) \cos (\theta)-M_{z}(y, z) \sin (\theta), \\
& M_{z}^{\prime}(y, z)=M_{z}(y, z) \cos (\theta)+M_{y}(y, z) \sin (\theta) .
\end{aligned}
$$

The magnetic field $B_{y, z}^{\prime}(y, z)$, which is produced by the distributions $M_{y, z}^{\prime}(y, z)$, is also calculated using Eqs. (2) and (3). After some algebraic transformations where expressions (4) and (5) have been inserted into the proper expressions for $B_{y, z}^{\prime}(y, z)$, we get

$$
B_{y}^{\prime}(y, z)=B_{y}(y, z) \cos (\theta)+B_{z}(y, z) \sin (\theta),
$$

$$
B_{z}^{\prime}(y, z)=B_{z}(y, z) \cos (\theta)-B_{y}(y, z) \sin (\theta) .
$$

By comparing these results with those of Eqs. (4) and (5), it is apparent that the magnetic field is turned through the opposite angle $-\theta$ without any change in its amplitude [1].

Next consider a Fourier transform of the vertical component of the magnetic field,

$$
\tilde{B}(p)=\int_{-\infty}^{\infty} B_{z}(y, z=0) \exp (\text { ipy }) d y .
$$

Substituting Eq. (3) into (8) and applying Cauchy's theorem, we may integrate with respect to $y$-variable analytically. As a result, we get the following expression for the Fourier transforms (8):

$$
\tilde{B}(p)=2 \pi|p| \int d y d z \exp (i p y) \exp (-|p z|)\left\{M_{z}(y, z)-i M_{y}(y, z) \operatorname{sgn}(p z)\right\} \text {. }
$$

The complex conjugation of expression (9) is equivalent to the change of the argument's sign,

$$
\tilde{B}(-p)=\tilde{B}^{*}(p),
$$

which is also evident from Eq. (8).

Let us consider now an infinitely long wiggler with the length of period $l$. We may expand its periodical magnetic field into the Fourier series with the following Fourier coefficients ( $k$ is integer):

$$
B_{z}(y, z=0)=\sum_{k=-\infty}^{\infty} \tilde{B}_{k} \exp \left(-i \frac{2 \pi k}{l} y\right),
$$

where

$$
\tilde{B}_{k}=\frac{1}{l} \int_{0}^{l} \exp \left(i \frac{2 \pi}{l} k y\right) B_{z}(y, z=0) d y .
$$

Let $b_{z}(y)$ be the vertical component of the magnetic field at the median plane $z=0$, which is produced by one wiggler's period. It follows from the concept of superposition that the wiggler's field is the sum of such oneperiod fields,

$$
B_{z}(y, z=0)=\sum_{n=-\infty}^{\infty} b_{z}(y+n l) .
$$

By substituting Eq. (13) into (12) and changing the variable $y=y^{\prime}-n l$, the sum of integrals passes into the integration over all values of $y^{\prime}$,

$$
\tilde{B}_{k}=\frac{1}{l} \int_{-\infty}^{\infty} \exp \left(i \frac{2 \pi}{l} k y^{\prime}\right) b_{z}\left(y^{\prime}\right) d y^{\prime} .
$$

As a consequence of Eqs. (9) and (14), we get the following expression for the Fourier coefficients:

$$
\tilde{B}_{k}=\frac{4 \pi^{2}|k|}{l^{2}} \int_{S} d y d z \exp \left(i \frac{2 \pi}{l} k y\right) \exp \left(-\frac{2 \pi}{l}|k z|\right)\left\{M_{z}(y, z)-i M_{y}(y, z) \operatorname{sgn}(k z)\right\},
$$

where the integration in (15) is performed over the cross section of this one period $S, k=0, \pm 1, \pm 2, \ldots$.

The expression (15) allows us to find the Fourier coefficients directly for wigglers with nonstandard designs, skipping an explicit calculation for wiggler magnetic fields, thus simplifying considerably the magnetic field analysis.

Let us apply the results obtained above for the infinitely long, pure permanent magnet planar wiggler with standard design. It is suggested that each of the top and bottom parts of the wiggler's period by itself involves $N$ rectangular uniformly magnetized blocks $(N=4$ for Fig. 1 and $N=$ 8 for Fig. 2). It has been assumed that from each block to the next, the easy axes are rotated by the angle $2 \pi / N$, with the first block being magnetized vertically and the following mirror-type symmetry about the median plane:

$$
M_{z}(y,-z)=M_{z}(y, z) \text { and } M_{y}(y,-z)=-M_{y}(y, z) .
$$

Relation (10) allows us to consider here only positive numbers of harmonic $k=1,2,3, \ldots$ For the $n$th magnetic block (both top and bottom), we get

$$
\begin{aligned}
M_{z}(y, z)-i M_{y}(y, z) & \operatorname{sgn}(z) \\
= & M \exp \left(-i \frac{2 \pi}{N}(n-1)\right),
\end{aligned}
$$

where $M=\sqrt{M_{z}^{2}+M_{y}^{2}}$ and is a constant, $\frac{l}{n}(n-1)<$ $y<\frac{l}{N} n$ and $n=1,2, \ldots, N$.

In this case, the integral in (15) may be broken down into the sum of $2 N$ integrals over the cross sections of the individual magnetic blocks with the following analytical evaluation of these individual integrals. The summation of the resulting series gives us 


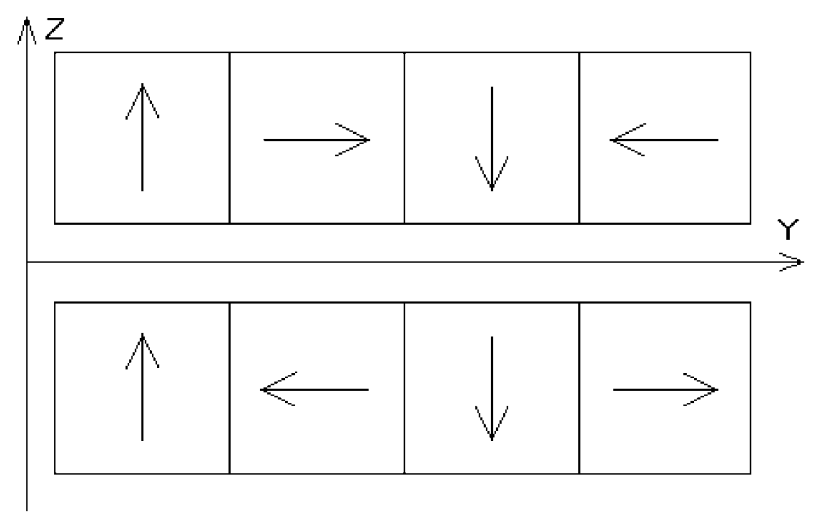

FIG. 1. Halbach wiggler configuration.

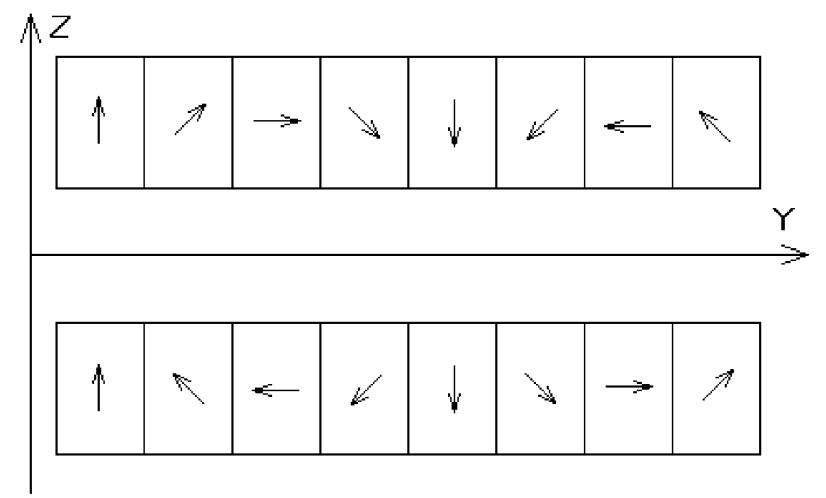

FIG. 2. Eight-blocks per period wiggler configuration.

$$
\tilde{B}_{k}=\frac{B_{r} N}{\pi k} \exp \left(-\frac{\pi g}{l} k\right)\left[1-\exp \left(-\frac{2 \pi h}{l} k\right)\right] \exp \left(\frac{i \pi}{N}\right) \sin \left(\frac{\pi}{N}\right)
$$

Here $k=1+m N, m=0,1,2, \ldots, h$ is the height of block, $g$ is the wiggler's gap, and $B_{r}=4 \pi M$ is a remanent field of the magnetic blocks. Substituting Eq. (18) into Eq. (11) with the use of (10), we get

$$
B_{z}(y, z=0)=2 \sum_{m=0}^{\infty}\left|\tilde{B}_{k}\right| \cos \left(\frac{2 \pi}{l} k y-\frac{\pi}{N}\right) .
$$

This formula is equivalent to that of a previous study [4] where it was derived through the direct field calculation.

The first harmonic (where $m=0$ and, correspondingly, $k=1$ ) has a maximum at the point with the longitudinal coordinate $y_{c}=l /(2 N)$ that is located at the middle of the vertically magnetized block. However, the correspondent arguments of cosine at this point for the succeeding nonzero harmonics (with $k=1+m N$ ) are equal to $m \pi$, and the contributions of the neighboring harmonics for the magnetic field value (19) are of opposite sign,

$$
\tilde{B}_{1}=\frac{4 \pi^{2}}{l^{2}} \int_{S} d y d z \exp \left[i\left(\frac{2 \pi}{l} y-\alpha(y, z)\right)\right] \exp \left(-\frac{2 \pi}{l}|z|\right) M(y, z) .
$$

It is well known that $\left|\int f(x) d x\right| \leq \int|f(x)| d x$. The equality is fulfilled if $|f(x)|=f(x)$. This relation, as applied to integral (22), implies that the integrand in (22) should be a real positive function. It means that the magnetic blocks should be magnetized as high as possible (though restricted by technical reasons), so that $M(y, z)=$ $M$ is a constant. In addition, the complex exponent in Eq. (22) should be equal to one, which gives

$$
\alpha(y, z)=\frac{2 \pi}{l} y .
$$

By this it is meant that the easy axis rotates continuously and progressively along the longitudinal coordinate, clockwise at the wiggler's top part and anticlockwise at the bottom part. In this case, the Fourier coefficients peak at $k= \pm 1$ and are equal to

$$
B_{z}\left(y_{c}, z=0\right)=2 \sum_{m=0}^{\infty}(-1)^{m}\left|\tilde{B}_{k}\right| .
$$

\section{UPPER THEORETICAL LIMIT FOR WIGGLER MAGNETIC FIELD}

Let us determine conditions in which the first harmonic of the magnetic field reaches its maximum. The expression in the braces of Eq. (15) may be presented as

$$
\begin{aligned}
& M_{z}(y, z)-i M_{y}(y, z) \operatorname{sgn}(z) \\
& =M(y, z) \exp (-i \alpha(y, z)),
\end{aligned}
$$

where $M(y, z)=\sqrt{M_{y}^{2}(y, z)+M_{z}^{2}(y, z)}$ is a real positive function. By substituting Eq. (21) into integral (15), we get at $k=1$

$$
\tilde{B}_{ \pm 1}^{\infty}=B_{r} \exp \left(-\frac{\pi g}{l}\right)\left[1-\exp \left(-\frac{2 \pi h}{l}\right)\right] .
$$

All higher harmonics of the wiggler magnetic field are equal to zero.

Let us now compare this theoretical limit with the first harmonic of the magnetic field produced by standard pure permanent magnet wigglers. For schemes described in Sec. II we have

$$
\tilde{B}_{1}=\tilde{B}_{1}^{\infty} \frac{N}{\pi} \exp \left(\frac{i \pi}{N}\right) \sin \left(\frac{\pi}{N}\right) .
$$

It follows from relation (25) that the Halbach-type wiggler configuration (Fig. 1) reaches $90 \%$ of the theoretical limit, and the configuration in Fig. 2 reaches $97.5 \%$ of the theoretical limit. This demonstrates that the performances of 
the standard schemes are close enough to the upper theoretical limit.

It should be noted that the wiggler with the configuration similar to the one in Fig. 2 (but with $N=6$ blocks per period) was suggested in Ref. [5].

\section{INCREASING THE MAGNETIC FIELD AMPLITUDE}

Let us examine the following transformation of the wiggler's magnetic system: we rotate the easy axes about the $x$ axis at each point of the wiggler's bottom part $(z<0)$ through the angle $\theta$ (i.e., in an anticlockwise direction) and at each point of the wiggler's top part $(z>0)$ through the opposite angle $-\theta$ (i.e., in a clockwise direction). This transformation is described by the equations

$$
\begin{aligned}
& M_{y}^{\prime}(y, z)=M_{y}(y, z) \cos (\theta)+M_{z}(y, z) \sin (\theta) \operatorname{sgn}(z), \\
& M_{z}^{\prime}(y, z)=M_{z}(y, z) \cos (\theta)-M_{y}(y, z) \sin (\theta) \operatorname{sgn}(z),
\end{aligned}
$$

from which it also follows that

$$
\begin{aligned}
M_{z}^{\prime}(y, z)-i M_{y}^{\prime}(y, z) & \operatorname{sgn}(p z) \\
= & {\left[M_{z}(y, z)-i M_{y}(y, z) \operatorname{sgn}(p z)\right] } \\
& \times \exp (-i \theta \operatorname{sgn}(p)) .
\end{aligned}
$$

The last expression shows that such modification of the magnetic system yields the additional phase factor in Fourier coefficients of the vertical magnetic field or in its Fourier transform [see Eq. (15) or Eq. (9), respectively] [6]

$$
\tilde{B}_{k}^{\prime}=\tilde{B}_{k} \exp (-i \theta \operatorname{sgn}(k))
$$

The shape of the magnetic field will be changed in the process since the phase factor differs at $k>0$ and $k<0$. At the same time, the absolute values of the Fourier coefficients will not be changed by this transformation.

The magnetic field, which is produced by this "rotated" configuration, is given by the Fourier series (11) with Fourier coefficients (29), where $\tilde{B}_{k}$ are determined by Eq. (18). From the above we might conclude that

$$
B_{z}^{\prime}(y, z=0)=2 \sum_{m=0}^{\infty}\left|\tilde{B}_{k}\right| \cos \left(\frac{2 \pi}{l} k y-\frac{\pi}{N}+\theta\right) .
$$

This expression peaks at $\theta=\frac{\pi}{N}$ and $y=0$, and its maximum value is equal to

$$
B_{z}^{\prime}(y=0, z=0)=2 \sum_{m=0}^{\infty}\left|\tilde{B}_{k}\right|,
$$

which is evidently higher than the value give by Eq. (20).

Figure 1 shows the standard Halbach configuration $(N=4)$, and Fig. 3 shows the configuration when the

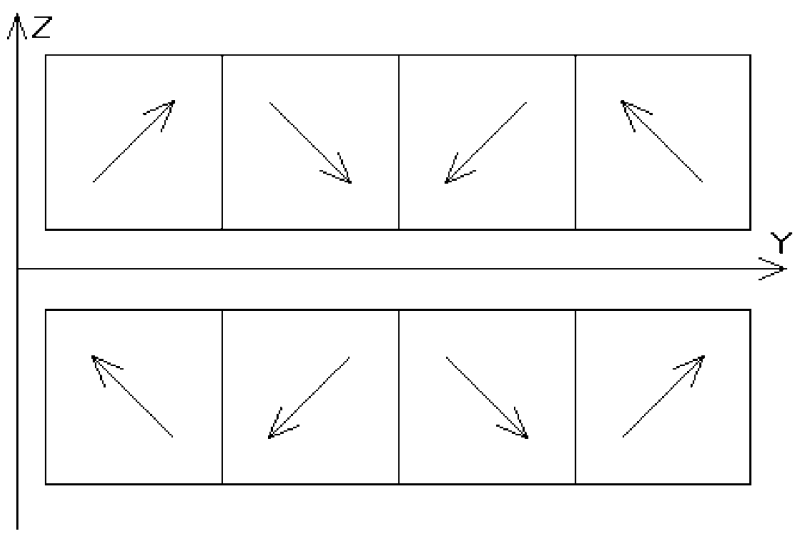

FIG. 3. Rotated wiggler configuration.

easy axes are properly rotated at the angle $\theta=0.25 \pi$. Figure 4 shows a comparison of the magnetic field profiles for these two configurations. The magnetic fields were calculated for the wigglers according to the following parameters: the length of period is $40 \mathrm{~mm}$, height of the magnetic blocks is $80 \mathrm{~mm}$, the gap is $2 \mathrm{~mm}$, and the remanent field of the magnetic blocks is $1.0 \mathrm{~T}$. As can be seen from these plots, the standard Halbach configuration generates the magnetic field with amplitude of only about $75 \%$ of the field amplitude, which is produced by the rotated configuration. The difference in the profiles results from the additional phase factor of the Fourier coefficients, with the same strength of field harmonics for both cases [see Eq. (29)].

Notice that an unusual arrangement for the wiggler magnets was suggested with the aim to enhance the gain on the third harmonic [7]. This design, with the magnetization vectors parallel and antiparallel to the wiggler axis, is just one example of the configurations (with $N=2$ and $\theta=0.5 \pi$, respectively) that can be achieved using this general approach.

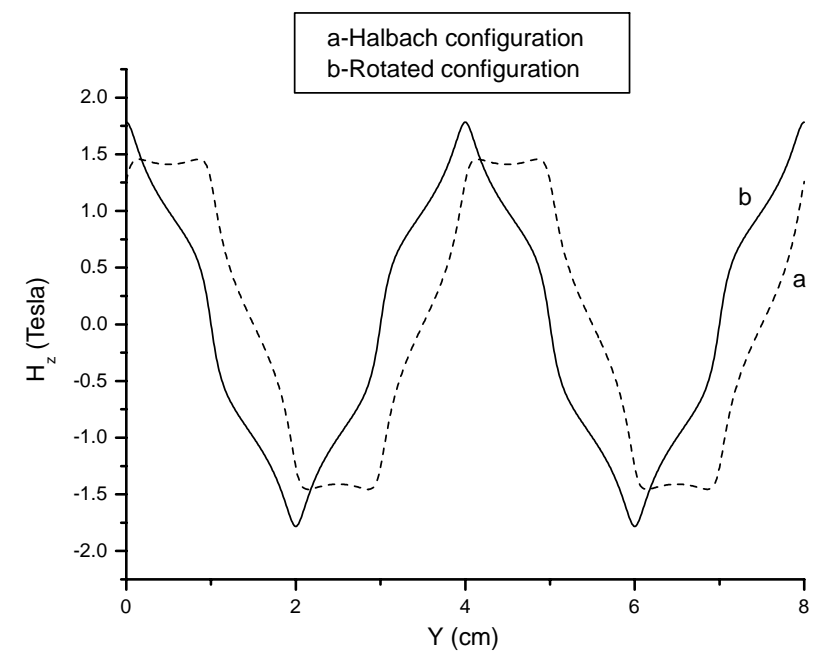

FIG. 4. Magnetic field produced by two different wiggler configurations - the Halbach and the rotated configurations. 


\section{PROPERTY OF UNIVERSALITY FOR PLANAR WIGGLERS}

The method considered here for analysis of the planar magnetic field allows us to state the following property of universality for planar wigglers, which is highly significant in many applications: if all easy axes of the wiggler's top part are rotated by the angle $\theta$ (anticlockwise) and at the same time all easy axes of its bottom part are rotated by the opposite angle $-\theta$ (clockwise) while the system geometry remains the same, the profile of the magnetic field varies, but the total power of wiggler radiation as well as the spectral positions of radiated harmonics remain unchanged by such modification. Notice that this transformation of the magnetization vectors that is described by Eqs. (26) and (27) differs from those described by the easy axis theorem [see Eqs. (4) and (5)] and, consequently, the results differ as well.

The total power of electromagnetic radiation, emitted by the high-energy electron in its passage through the wiggler's magnetic field, is equal to [3]

$$
W=\frac{2 e^{4} \gamma^{2}}{3 m^{2} c^{4}} \int_{L} B_{z}^{2}(y, z=0) d y,
$$

where $L$ is the wiggler's total length, $c$ is the speed of light, $e$ and $m$ are the electron charge and mass, respectively, and $\gamma$ is its reduced energy. Due to the periodicity of the magnetic field, the integral in (32) is equal to the integral over one period $l$ multiplied by the number of periods $L / l$. The one-period integral of the magnetic field squared, according to Parceval's theorem, is proportional to the sum of the field's Fourier coefficients, raised to the second power. As a result, we get

$$
W=\frac{2 e^{4} \gamma^{2}}{3 m^{2} c^{4}} L \sum_{k=-\infty}^{\infty}\left|\tilde{B}_{k}\right|^{2} .
$$

Since the transformation, which is given by Eqs. (26) and (27), does not change the absolute values of the Fourier coefficients [see relation (29)], the total power (32) is invariant under the considered transformation.

The spectral positions of the wiggler's harmonics are also invariant. The wavelength $\lambda_{n}$ of the $n$th harmonic on the wiggler's axis is given by

$$
\lambda_{n}=\frac{l}{2 \gamma^{2} n}\left(1+0.5 K^{2}\right),
$$

where $K$ is the wiggler's deflection parameter and generally is determined by the following relation:

$$
K^{2}=\left(\frac{e l}{\pi m c^{2}}\right)^{2} \sum_{k=1}^{\infty} \frac{\left|\tilde{B}_{k}\right|^{2}}{k^{2}} .
$$

Similarly to the total power, the invariance of the absolute values of the Fourier coefficients causes the invariance of the deflection parameter and, consequently, the wavelengths of the generated harmonics [6].

Let us discuss briefly how the rotation of the magnetization vectors [given by Eqs. (26) and (27)] affects the intensities of the radiated harmonics. For undulators with a relatively weak magnetic field (i.e., in the case of a small deflection parameter, when the radiation is of a dipole-type in the electron rest frame), the intensities of the undulator's harmonics are determined by the magnetic field Fourier transform squared [8]. Consequently, the spectral characteristics of dipole radiation remain also invariant under this transformation process of the magnetic system.

For planar wigglers with a relatively strong magnetic field $K \geq 1$, generally this transformation of the wiggler's magnetization will change the intensities of radiated harmonics, increasing some of them and decreasing others in such a way that the total power remains the same. Two wigglers with standard design (Fig. 1) and with magnetization vectors rotated through $0.25 \pi$ (Fig. 3), having parameters described in the previous section, were taken as an example. The deflection parameter is equal to $K=$ 5.75 for both cases giving reasonably intensive high harmonics. On-axis intensities of a few harmonics, generated by $1 \mathrm{GeV}$ electron beam were numerically simulated for these different configurations. These simulations show that the intensity of the first harmonic in the second (rotated) case is lower by $0.4 \%$ as compared with the standard wiggler, while the intensities of the third, fifth, and seventh harmonics are higher by $17 \%, 8 \%$, and $4 \%$, respectively. The on-axis intensities of ninth and higher harmonics in rotated wigglers are again lower than those of the standard configuration.

[1] K. Halbach, Nucl. Instrum. Methods 169, 1 (1980).

[2] B. Diviacco and R.P. Walker, Nucl. Instrum. Methods Phys. Res., Sect. A 292, 517 (1990).

[3] L. D. Landau and E. M. Lifshitz, in The Classical Theory of Fields (Pergamon, New York, 1975), 4th ed.

[4] K. Halbach, Nucl. Instrum. Methods 187, 109 (1981).

[5] G. Rakowsky, B. Bobbs, R. Burke, W. McMullin, and G. Swoyer, Nucl. Instrum. Methods Phys. Res., Sect. A 296, 597 (1990).

[6] M. N. Smolyakov, Nucl. Instrum. Methods Phys. Res., Sect. A 448, 85 (2000).

[7] P. G. O'Shea, S. C. Bender, D. A. Byrd, J. W. Early, D. W. Feldman, C. M. Fortgang, J. C. Goldstein, B. E. Newnam, R. L. Sheffield, R. W. Warren, and T. J. Zaugg, Nucl. Instrum. Methods Phys. Res., Sect. A 341, 7 (1994).

[8] R. Coisson, Phys. Rev. A 20, 524 (1979). 